\title{
Orbit Computation in Celestial Mechanics by Urabe's Method
}

By

\author{
René VAN Dooren*
}

\begin{abstract}
The numerical computation of orbits in celestial mechanics can be performed by applying Urabe's method for the Galerkin procedure. High order Galerkin approximations are computed for two examples: Hill's variation orbit of the moon and the orbit of an artificial earth satellite. Excellent agreement with known results is obtained.
\end{abstract}

\section{Introduction}

In [1-2] Urabe introduced a complete criterion for the study of periodic solutions of certain periodic non-linear ordinary differential equations. This procedure permits to compute high order Galerkin approximations with a very high precision by applying Newton's iterative method and using an electronic computer, to discuss the existence and the stability of an exact isolated periodic solution in a small neighborhood of a numerical computed Galerkin approximation and to determine the error bound of this Galerkin approximation. Urabe [2-3] applied this method to a highly nonlinear equation which had been first studied by Cesari [4] using a different existence analysis more topological in character, to a weakly non-linear van der Pol equation, and also to a Duffing equation in order to determine a subharmonic solution. Bouc [5] reexamined these examples under a different viewpoint taking into account the symmetry of the considered equations. In [6-10] we applied Urabe's complete method to coupled Duffing equations with two degrees of freedom. In all these examples the right

Communicated by S. Hitotumatu, March 9, 1973.

* Department of Rational Mechanics, Faculty of Applied Sciences, Free University of Brussels, Belgium. 
hand side of the considered system $\ddot{x}=X(x, \dot{x}, t)$ is a polynomial in $x$ and $\dot{x}$ with periodic coefficients.

The purpose of this study is to show that high order Galerkin approximations can be obtained without difficulties by Urabe's method when $X(x, \dot{x}, t)$ has no longer the mentioned properties. This is especially the case for perturbed Keplerian motion in celestial mechanics. As an illustration we compute Galerkin approximations of high order for Hill's variation orbit of the moon and for the orbit of an artificial earth satellite. The numercial results are in excellent agreement with known results obtained by Hill $[11-12]$.

\section{Galerkin Approximations by Urabe's Method}

We consider a real periodic differential system of the form

$$
\ddot{x}=X(x, \dot{x}, t)
$$

where $x, \dot{x}$ and $X(x, \dot{x}, t)$ are vectors of the same dimension and $X(x$, $\dot{x}, t)$ is periodic in $t$ with period $2 \pi$. A dot means differentiation with respect to $t$. We seek an approximate periodic solution of (2.1) with period $2 \pi$ represented by a trigonometric polynomial of the form

$$
x_{m}(t)=a_{0}+\sum_{n=1}^{m}\left(a_{2 n-1} \sin n t+a_{2 n} \cos n t\right) .
$$

The unknown coefficients $a_{\nu}$ are determined by a balance procedure applied to the following equation

$$
\ddot{x}_{m}(t)=X_{m}\left(x_{m}(t), \dot{x}_{m}(t), t\right)
$$

in which $X_{m}\left(x_{m}(t), \dot{x}_{m}(t), t\right)$ represents the Fourier series of $X\left(x_{m}(t)\right.$, $\left.\dot{x}_{m}(t), t\right)$ truncated after the harmonics of order $m$

$$
X_{m}\left(x_{m}(t), \dot{x}_{m}(t), t\right)=A_{0}+\sum_{n=1}^{m}\left(A_{2 n-1} \sin n t+A_{2 n} \cos n t\right)
$$

The Fourier coefficients $A_{\nu}$ are given by

$$
A_{0}=\frac{1}{2 \pi} \int_{0}^{2 \pi} X\left[x_{m}(s), \dot{x}_{m}(s), s\right] d s
$$




$$
\begin{gathered}
A_{2 n-1}=\frac{1}{\pi} \int_{0}^{2 \pi} X\left[x_{m}(s), \dot{x}_{m}(s), s\right] \sin n s d s \\
A_{2 n}=\frac{1}{\pi} \int_{0}^{2 \pi} X\left[x_{m}(s), \dot{x}_{m}(s), \mathrm{s}\right] \cos n s d s \\
n=1,2, \ldots, m .
\end{gathered}
$$

Equating the coefficients of $1, \sin n t$ and $\cos n t$ with $n=1,2, \ldots, m$ in (2.3), we obtain the determining equations for the coefficients $a_{\nu}$

$$
\begin{aligned}
F_{0}(\alpha) & \equiv \frac{1}{2 \pi} \int_{0}^{2 \pi} X\left[x_{m}(s), \dot{x}_{m}(s), s\right] d s \\
F_{2 n-1}(\alpha) & \equiv \frac{1}{\pi} \int_{0}^{2 \pi} X\left[x_{m}(s), \dot{x}_{m}(s), s\right] \sin n s d s+n^{2} a_{2 n-1}=0 \\
F_{2 n}(\alpha) & \equiv \frac{1}{\pi} \int_{0}^{2 \pi} X\left[x_{m}(s), \dot{x}_{m}(s), s\right] \cos n s d s+n^{2} a_{2 n}=0
\end{aligned}
$$

with $n=1,2, \ldots, m$ and $\alpha \equiv\left(a_{0}, a_{1}, \ldots, a_{2 m-1}, a_{2 m}\right)$.

This method to determine the coefficients of the approximate solution (2.2) of the system (2.1) is the Galerkin method (see also [13-17]). The trigonometric polynomials (2.2) are called Galerkin approximations of order $m$. The equations which determine the coefficients $a_{\nu}$ can be rewritten as follows

$$
F_{\mu}(\alpha)=0 \quad \mu=0,1, \ldots, 2 m
$$

Suppose $\bar{\alpha} \equiv\left(\bar{a}_{0}, \bar{a}_{1}, \ldots, \bar{a}_{2 m-1}, \bar{a}_{2 m}\right)$ is an approximate solution of (2.7) and let us apply Newton's iterative method to solve these non-linear equations. Then for the imposed approximate solution $\bar{\alpha}$, we have

$$
F_{\mu}(\bar{\alpha})+\sum_{\nu=0}^{2 m} J_{\mu \nu}(\bar{\alpha}) h_{\nu}=0 \quad \mu=0,1, \ldots, 2 m
$$

where

$$
\begin{gathered}
h_{\nu}=a_{\nu}-\bar{a}_{\nu} \quad J_{\mu \nu}(\bar{\alpha})=\frac{\partial F_{\mu}}{\partial a_{\nu}}(\bar{\alpha}) \\
\mu, \nu=0,1, \ldots, 2 m .
\end{gathered}
$$

Urabe [2] described a numerical approximation method to calculate the 
values of $F_{\mu}$ and their partial derivatives $\partial F_{\mu} / \partial a_{\nu}$ which occur in Newton's iterative method, when the values of an approximate solution $\bar{\alpha}$ of (2.7) are given. This approximation method is based on the computation of the Fourier coefficients $A_{\nu}$ in (2.5) by the following approximation formulae

$$
\begin{aligned}
A_{0} & =\frac{1}{2 N} \sum_{i=1}^{2 N} X\left[x_{m}\left(t_{i}\right), \dot{x}_{m}\left(t_{i}\right), t_{i}\right] \\
A_{2 n-1} & =\frac{1}{N} \sum_{i=1}^{2 N} X\left[x_{m}\left(t_{i}\right), \dot{x}_{m}\left(t_{i}\right), t_{i}\right] \sin n t_{i} \\
A_{2 n} & =\frac{1}{N} \sum_{i=1}^{2 N} X\left[x_{m}\left(t_{i}\right), \dot{x}_{m}\left(t_{i}\right), t_{i}\right] \cos n t_{i}
\end{aligned}
$$

with $\quad n=1,2, \ldots, m \quad N>m$

$$
t_{i}=\frac{2 i-1}{2 N} \pi \quad i=1,2, \ldots, 2 N
$$

For further details we refer to [2].

By solving numerically the linear equations (2.8) with respect to the unknowns $h_{\nu}$ we obtain the values of the coefficients $a_{\nu}$. The starting values in Newton's iterative method are found either by applying the balance method of the first harmonics or by using the known results of a slightly different system.

\section{Hill's Variation Orbit}

Hill's equations for the motion of the moon in a rotating coordinate system can be written as $[11-12]$

$$
\begin{array}{ll}
\ddot{x}_{1}-2 \varepsilon \dot{x}_{2}+\left(\frac{\lambda}{r^{3}}-3 \varepsilon^{2}\right) x_{1} & =0 \\
\ddot{x}_{2}+2 \varepsilon \dot{x}_{1}+\frac{\lambda}{r^{3}} x_{2} & =0
\end{array}
$$

with

$$
r=\left(x_{1}^{2}+x_{2}^{2}\right)^{1 / 2} \text { and } \lambda=(1+\varepsilon)^{2} / \beta^{3} \text {. }
$$

Hill obtained a trigonometric series solution and calculated a numerical 
solution to fifteen decimal places with the following values of the parameters $\varepsilon$ and $\beta$

$$
\varepsilon=0.080848933808312 \text { and } \beta=0.999093141975298 \text {. }
$$

The numerical results are

$$
\begin{aligned}
x_{1}(t) & =0.991304253038460 \cos t+0.001515871270049 \cos 3 t \\
& +0.000005881116971 \cos 5 t+0.000000030043916 \cos 7 t \\
& +0.000000000175332 \cos 9 t+0.000000000001107 \cos 11 t \\
& +0.000000000000007 \cos 13 t \\
x_{2}(t) & =1.008695746961540 \sin t+0.001515543689077 \sin 3 t \\
& +0.000005876196185 \sin 5 t+0.000000030019348 \sin 7 t \\
& +0.000000000175204 \sin 9 t+0.000000000001107 \sin 11 t \\
& +0.000000000000007 \sin 13 t .
\end{aligned}
$$

Now let us apply Urabe's method to obtain Hill's variation orbit. The equations (3.1) can be rewritten as follows

$$
\begin{aligned}
& \ddot{x}_{1}=X_{1}\left(x_{1}, x_{2}, \dot{x}_{2}\right) \\
& \ddot{x}_{2}=X_{2}\left(x_{1}, x_{2}, \dot{x}_{1}\right)
\end{aligned}
$$

with

$$
\begin{aligned}
& X_{1}\left(x_{1}, x_{2}, \dot{x}_{2}\right)=2 \varepsilon \dot{x}_{2}-\left(\lambda r^{-3}-3 \varepsilon^{2}\right) x_{1} \\
& X_{2}\left(x_{1}, x_{2}, \dot{x}_{1}\right)=-2 \varepsilon \dot{x}_{1}-\lambda r^{-3} x_{2}
\end{aligned}
$$

Although $X_{1}$ and $X_{2}$ do not contain $t$ explicitly, we may look for approximate periodic solutions of (3.5) with period $2 \pi$ represented by trigonometric polynomials of the form

$$
\begin{aligned}
& x_{1}(t)=a_{0}+\sum_{n=1}^{m}\left(a_{2 n-1} \sin n t+a_{2 n} \cos n t\right) \\
& x_{2}(t)=b_{0}+\sum_{n=1}^{m}\left(b_{2 n-1} \sin n t+b_{2 n} \cos n t\right)
\end{aligned}
$$

where the unknown coefficients $a_{\nu}$ and $b_{\nu}$ should be determined by Urabe's method for the Galerkin procedure as described in Section 2. The starting 
values in Newton's iterative method are taken as follows

$$
\begin{array}{llll}
\bar{a}_{0}=0 & \bar{a}_{1}=0 & \bar{a}_{2}=1 & \bar{a}_{3}=\bar{a}_{4}=\cdots=\bar{a}_{2 m}=0 \\
\bar{b}_{0}=0 & \bar{b}_{1}=1 & \bar{b}_{2}=0 & \bar{b}_{3}=\bar{b}_{4}=\cdots=\bar{b}_{2 m}=0
\end{array}
$$

The computations carried out on the computer CDC 6400 at the University of Brussels, yield the following Galerkin approximations of order $m=13$ by Urabe's method with $N=35$ and a required precision of 14 decimal digits for the coefficients $a_{\nu}$ and $b_{\nu}$ obtained after 4 iterations

$$
\begin{aligned}
x_{1}(t) & =0.99130425303848 \cos t+0.00151587127005 \cos 3 t \\
& +0.00000588111697 \cos 5 t+0.00000003004392 \cos 7 t \\
& +0.00000000017533 \cos 9 t+0.00000000000111 \cos 11 t \\
& +0.00000000000001 \cos 13 t \\
x_{2}(t) & =1.00869574696158 \sin t+0.00151554368908 \sin 3 t \\
& +0.00000587619619 \sin 5 t+0.00000003001935 \sin 7 t \\
& +0.00000000017520 \sin 9 t+0.00000000000111 \sin 11 t \\
& +0.00000000000001 \sin 13 t .
\end{aligned}
$$

Compared to Hill's results (3.4) we notice an excellent agreement (about 13 decimal digits).

\section{Artificial Satellite of the Earth}

The differential equations of the motion of an artificial earth satellite perturbed by the dominant oblateness term of the earth are [18]

$$
\begin{aligned}
& \ddot{x}_{1}=-\frac{x_{1}}{r^{3}}+k\left(\frac{5 x_{1} x_{3}^{2}}{r^{7}}-\frac{x_{1}}{r^{5}}\right) \\
& \ddot{x}_{2}=-\frac{x_{2}}{r^{3}}+k\left(\frac{5 x_{2} x_{3}^{2}}{r^{7}}-\frac{x_{2}}{r^{5}}\right) \\
& \ddot{x}_{3}=-\frac{x_{3}}{r^{3}}+k\left(\frac{5 x_{3}^{3}}{r^{7}}-\frac{3 x_{3}}{r^{5}}\right)
\end{aligned}
$$

with

$$
r=\left(x_{1}^{2}+x_{2}^{2}+x_{3}^{2}\right)^{1 / 2}
$$


In the determination of the parameter $k$ only the dominant oblateness term has been taken into account.

Let us assume that the unperturbed motion $(k=0)$ is circular

$$
\begin{aligned}
& x_{1}(t)=\cos t \\
& x_{2}(t)=\cos i \sin t \\
& x_{3}(t)=\sin i \sin t
\end{aligned}
$$

where $i$ is the inclination of the orbit of the satellite.

Then following Urabe's method we can seek approximate periodic solutions with period $2 \pi$ of the perturbed motion described by (4.1) which are represented by truncated Fourier series. The starting values in the iterative procedure are taken from the known solution (4.3) of the unperturbed motion. The following values of the parameters $i$ and $k$ are adopted

$$
i=\frac{\pi}{2} \quad k=0.0014
$$

The selected value of $k$ corresponds to an altitude of the satellite of approximately $492 \mathrm{~km}$ above the surface of the earth at perigee.

The Galerkin approximations of order $m=9$ obtained after 4 iterations with $N=25$ and a precision of 14 decimal digits for the unknown coefficients, are as follows

$$
\begin{aligned}
x_{1}(t) & =0.99982458758998 \cos t+0.00017515869991 \cos 3 t \\
& -0.00000001878087 \cos 5 t+0.00000000000276 \cos 7 t \\
& +0.00000000000000 \cos 9 t \\
x_{2}(t) & =0.00000000000000 \sin t+0.00000000000000 \sin 3 t \\
& +0.00000000000000 \sin 5 t+0.00000000000000 \sin 7 t \\
& +0.00000000000000 \sin 9 t \\
x_{3}(t) & =0.99970759298578 \sin t+0.00017516895755 \sin 3 t \\
& -0.00000001878254 \sin 5 t+0.00000000000276 \sin 7 t \\
& +0.00000000000000 \sin 9 t .
\end{aligned}
$$

The two examples clearly show that Galerkin approximations of high order for perturbed Keplerian motion in celestial mechanics can be computed with a very high precision by applying Urabe's method for the Galerkin procedure. 


\section{References}

[1] Urabe M., Galerkin's procedure for nonlinear periodic systems, Arch. Rat. Mech. Anal. 20, 120-152 (1965).

[2] Urabe, M. and Reiter, A., Numerical computation of nonlinear forced oscillations by Galerkin's procedure, J. of Math. Anal. Appl. 14, 107-140 (1966).

[ 3 ] Urabe, M., Numerical investigation of subharmonic solutions to Duffing's equation, Publ. RIMS Kyoto Univ. 5, 79-112 (1969).

[4] Cesari, L., Functional analysis and periodic solutions of nonlinear differential equations, Contrib. Diff. Eqs. 1, 149-187 (1963).

[5] Bouc, R., Sur la méthode de Galerkin-Urabe pour les systèmes différentiels périodiques, Int. J. Non-linear Mech. (to appear).

[6] Van Dooren, R., Recherche numérique d'oscillations composées du type additif dans un système oscillant non linéaire amorti à deux degrés de liberté, Académie Royale de Belgique, Classe des Sciences, Tome LVII, 524-544 (1971).

[7] Van Dooren, R., Harmonic vibrations and combination tones of summed type in forced non-linear mechanical systems (in Netherlands), Doctor Thesis, Free University of Brussels 1971.

[8] Van Dooren, R., Numerical computation of forced oscillations in coupled Duffing epuations, Num. Math. 20, 300-311 (1973).

[9] Van Dooren, R., An analytical method for certain weakly non-linear periodic differential systems, Académie Royale de Belgique. Classe des Sciences (to appear).

[10] Van Dooren, R., Forced oscillations in coupled Duffing equations by an analytical method of varying amplitudes and phase angles, Koninklijke Vlaamse Academie van België. 34, $\mathrm{n}^{\circ} 2$ (1972).

[11] Hill, G. W., Researches in the lunar theory, Am. J. Math. 1 (1878).

[12] Brouwer, D. and Clemence, G. M., Methods of celestial mechanics, Academic Press 1961, p. 336.

[13] Kantorovich, L.V. and Krylov, V.I., Approximate methods of higher analysis, Groningen. Noordhoff 1964, p. 258.

[14] Cesari, L., Functional analysis and Galerkin's method. Michigan, Math. J. 11, 385-414 (1964).

[15] Urabe, M., Periodic solutions of differential systems, Galerkin's procedure and the method of averaging, Journal of differential equations 2, 265-280 (1966).

[16] Urabe, M., Galerkin's procedure for nonlinear periodic systems and its extension to multi-point boundary value problems for general nonlinear systems. In: Numerical solutions of nonlinear differential equations, Proceedings of an Advanced Symposium, University of Wisconsin 1966; New York, Wiley 1966, p. 297.

[17] Bouc, R., Remarque sur un résultat d'Urabe, Int.J. Non-linear Mech. 3, 99-111 (1968).

[18] Stiefel, E. and Bettis, D.G., Stabilization of Cowell's method, Num. Math. 13, 154-175 (1969). 